\title{
Nerve-Sparing Radical Hysterectomy Using the Okabayashi-Kobayashi Method
}

\author{
Noriaki Sakuragi, MD, PhD ${ }^{1,2}$ Masanori Kaneuchi, MD, PhD ${ }^{1}$ \\ ${ }^{1}$ Center for Women's Health (Gynecology), Otaru General Hospital, \\ Address for correspondence Noriaki Sakuragi, MD, PhD, Center for \\ Otaru, Japan \\ 2 Professor Emeritus, Department of Obstetrics and Gynecology, \\ Women's Health, Otaru General Hospital, Wakamatsu 1-1-1, Otaru, \\ 047-8550 Japan (e-mail: nsakuragi@jcom.home.ne.jp).
} Hokkaido University Graduate School of Medicine, Sapporo,

Hokkaido, Japan

Surg J (NY) 2021;7(suppl S2):S48-S56.

\begin{abstract}
Keywords

- fascia

- paracervical tissues

- paracolpium

- tissue plane

- traction and counter traction

Radical hysterectomy $(\mathrm{RH})$ is a standard treatment for early-stage cervical cancer. This surgery extirpates the uterus along with the paracervical tissues, vagina, and the paracolpium to achieve local control. Pelvic lymphadenectomy is a critical component of $\mathrm{RH}$ performed for regional control. A clear understanding of pelvic anatomy is critical to safely performing a $\mathrm{RH}$ and achieving optimal oncological and functional outcomes. The various surgical steps can damage the pelvic autonomic nerves, and a systematic nerve-sparing technique is used for the preservation of autonomic nerves. There is an intricate vascular network in the lateral paracervix (cardinal ligament) and the pelvic sidewall. We need to comprehend the three-dimensional structure of the vascular and nerve anatomy in the pelvis to perform RH effectively and safely. We can create six spaces around the uterine cervix, including the paravesical spaces, pararectal spaces, a vesicovaginal space, and a rectovaginal space to reveal the target of extirpation. It is critical to find the proper tissue plane separated by the layers of membranous connective tissue (fascia), in order to minimize intraoperative bleeding.
\end{abstract}

\section{Radical Hysterectomy Methods}

The method of radical hysterectomy (RH) commonly used in Western countries is the Wertheim operation modified by Meigs. ${ }^{1}$ The method used in Japan is the Okabayashi operation. ${ }^{2}$ The Okabayashi and Latzko methods ${ }^{3}$ are similar in that they create paravesical and pararectal spaces to separate the three components (ventral, lateral, and dorsal parts) of the parametrial/paracervical tissue ( $\mathbf{- F i g . ~ 1 , ~ r e d - c o l o r e d ~ a r e a ) . ~}$ The Okabayashi method uniquely identifies the paravaginal space after the excision of the anterior layer of the vesicouterine ligament. In this report, we use the term vesicouterine ligament to indicate the anterior layer of the vesicouterine ligament and the term vesicovaginal ligament to denote the posterior layer of the vesicouterine ligament. With this meth-

published online

December 1, 2021
DOI https://doi.org/ 10.1055/s-0041-1739119. ISSN 2378-5128. od, the paravaginal space connecting to the paravesical space is developed to dissect the vesicovaginal ligament. This procedure results in the extensive excision of the vagina and paracolpium, conferring high radicality to the surgery. Kobayashi modified the Okabayashi RH with the aim of preserving the pelvic nerves. ${ }^{4,5}$ In this article, we described the nerve-sparing Okabayashi-Kobayashi method of RH. ${ }^{6,7}$

\section{Preoperative Workup}

* Pathological diagnosis of invasive carcinoma.

* Bimanual examination.

Vaginal invasion: abnormal resistance on the vaginal fornices. (c) 2021. The Author(s).

This is an open access article published by Thieme under the terms of the Creative Commons Attribution-NonDerivative-NonCommercial-License, permitting copying and reproduction so long as the original work is given appropriate credit. Contents may not be used for commercial purposes, or adapted, remixed, transformed or built upon. (https://creativecommons.org/ licenses/by-nc-nd/4.0/)

Thieme Medical Publishers, Inc., 333 Seventh Avenue, 18th Floor, New York, NY 10001, USA 


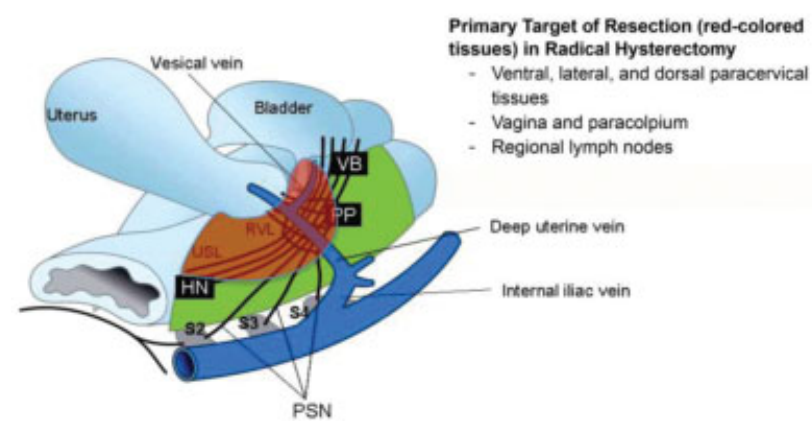

Fig. 1 The target of resection in radical hysterectomy $(\mathrm{RH})$ includes the cardinal ligament (lateral paracervix), the vesicouterine/vesicovaginal ligament (the ventral paracervix), the uterosacral/rectovaginal ligament (the dorsal paracervix), and the vagina/paracolpium.

Parametrial invasion: abnormal resistance of the paracervix is an alarming sign for parametrial invasion in deep infiltrating tumors, even if it is not nodular resistance.

* Imaging

Evaluation of tumor extension (size, depth, extracervical invasion).

$\square$ Extrapelvic extension and distant metastasis.

\section{Surgical Steps}

1) Laparotomy and preparation of the operative field. $\downarrow$

2) Opening the retroperitoneal space.

$\downarrow$

3) Pelvic lymphadenectomy (up to the obturator nerve).

$\downarrow$

4) Separation of the bladder and preparation of the uterine artery.

$\downarrow$

5) Removing the lymph nodes around the uterine artery and the cardinal ligament nodes below the obturator nerve.

$\downarrow$

6) Separation of the ureter and the transection of the uterine artery (at the origin from the internal iliac artery or medial to the ureter to preserve the ureteral branch of the uterine artery). $\downarrow$

7) Dissection and transection of the vesicouterine ligament (the anterior layer of the vesicouterine ligament in some textbooks). $\downarrow$

8) Separation of the hypogastric nerve and transection of the uterosacral ligament.

$\downarrow$
9) Dissection and transection of the vesicovaginal ligament (the posterior layer of the vesicouterine ligament in some textbooks).

$\downarrow$

10) Transection of the deep uterine vein (vascular portion of the cardinal ligament).

$\downarrow$

11) Selective transection of the uterine branches of the pelvic plexus (the inferior hypogastric plexus) and preservation of the pelvic plexus and its vesical branches.

$\downarrow$

12) Transection of the remaining rectovaginal ligament.

$\downarrow$

13) Amputation of the vagina/paracolpium and the suture.

$\downarrow$

14) Dissection of the common iliac nodes and presacral nodes.

$\downarrow$

15) Ovarian transposition to the paracolic gutter.

$\downarrow$

16) Peritoneal and abdominal closure.

\section{Patient Selection}

The evaluation of the applicability of a RH for a patient with cervical cancer is critical. We must determine the tumor size and the presence or absence of the tumor's extension outside the uterine cervix. Nerve-sparing RH is recommended for a patient with 2018 International Federation of Gynecology and Obstetrics stage IB1 $(\leq 2 \mathrm{~cm})$, IB2 $(\leq 4 \mathrm{~cm})$, and IIA1 $(\leq 4 \mathrm{~cm})$ cervical cancer. $\mathrm{RH}$ can be indicated for selected patients with stage IB3 $(>4 \mathrm{~cm})$ and IIA2 cervical cancer. Stage IIB disease may be an indication for radical hysterectomy in some countries, depending on the tumor's extent, the surgeon's expertise, and the institution's experience. The selection of patients who would benefit from surgical treatment is essential. Diagnosis of stage IIB disease is made clinically using the bimanual examination. Resistance in the parametrium/paracolpium suggests tumor invasion; however, some benign lesions such as endometriosis and inflammation may mimic the malignant infiltration. If there is nodular resistance, it may indicate lymph node metastasis. Clinical diagnosis of parametrium and paracolpium invasion is limited by its reduced sensitivity and reproducibility. Magnetic resonance imaging (MRI) can help improve the accuracy of diagnosis.

\section{Laparotomy and Operative Field}

The abdominal cavity is opened through a median incision, and a retractor system is used to obtain an adequate exposure of the lower abdominal cavity and a proper operative field. Careful inspection of the pelvic and abdominal cavity is 
required, and any adhesions should be released sharply to restore the pelvic organs' normal anatomical position. Two straight Pean clamps are used to hold the uterus. Pean clamps are placed closely on the lateral side of the uterus. Appropriate traction of the uterus is needed during the entire $\mathrm{RH}$ procedure to provide adequate tension on the tissues to be dissected. The uterus should be pulled upward and in the opposite direction of the surgical procedure being performed.

\section{Opening the Retroperitoneal Space}

The landmark for safely entering the retroperitoneal space is the external iliac artery. The round ligament is clamped and pulled upward. The stretched peritoneum of the anterior leaf of the broad ligament is incised just below the round ligament, and the incision is extended cranially. We can observe the membranous layers composed of the peritoneum, subperitoneal fascia, loose areolar tissues, and the fascial structures enveloping the vasculature and ureter. The retroperitoneal space is opened and developed using blunt dissection by paying attention to the membranous fascia (-Fig. 2). Sharp dissection using Kelly forceps and scissors, or electrocautery is also used when suitable. Correct tissue plane dissection will cause less bleeding.

The ureter crosses the vascular triangle composed of the external iliac, the internal iliac, and the common iliac arteries obliquely from the outside to the inside (-Fig. 3 ). The ureter is moved medially, and the connective tissue along the ventral surface of the internal iliac artery and the umbilical artery, which is an essential landmark for the pararectal and the paravesical spaces, can be dissected. The pararectal space's entrance filled with loose areolar tissue (pararectal space, so-called Latzko space) is located by medial displacement of the ureter and the hypogastric nerve, and between the internal iliac artery and the ureter. The dissection of the loose areolar tissue should be performed to avoid damage to the internal iliac vein and its branches. Strong force should not be necessary to open the tissue plane with loose areolar tissue. However, bleeding may be difficult to control if these veins are injured at this stage with incomplete preparation of

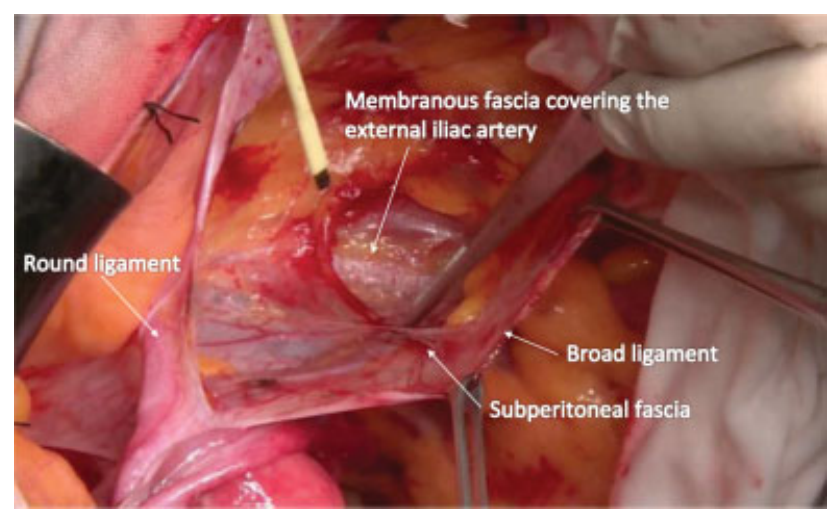

Fig. 2 The membranous structures enveloping the pelvic organs should be considered when opening the retroperitoneal space and dissecting the tissues and organs.

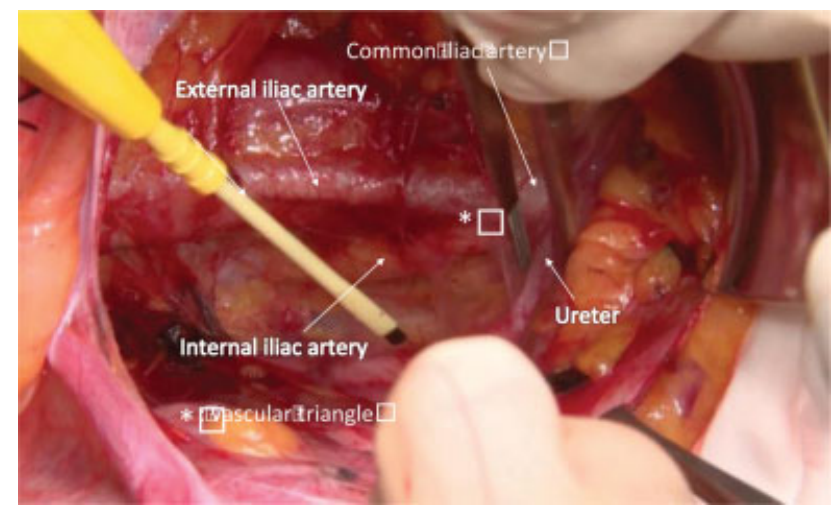

Fig. 3 The vascular triangle serves as the landmark to identify the ureter. Both the vascular system and the ureter are enveloped by different membranous fascia.

the paracervical tissues. To open the pararectal space, the ureter and the hypogastric nerve should be displaced medially with the intension of leaving only the uterosacral or rectovaginal ligament itself. The axis of the space should be along the curvature of the sacrum. The pararectal space is fenced by the internal iliac vessels laterally, the rectum medially, and the uterine artery and veins caudally (-Fig. 4). Another way to enter the pararectal space is to separate the ureter and the hypogastric nerve as a component enveloped by the ureterohypogastric fascia from the posterior leaf of the broad ligament and the lateral side of the rectum (pararectal space, so-called Okabayashi space). The bottom of the Latzko space and the Okabayashi space share a common space after removing the thin membranous tissue below the hypogastric nerve.

The paravesical space is opened between the umbilical artery and the external iliac vein. This space is filled with loose areolar tissues. Space is developed using blunt dissection toward the pelvic floor ( $\mathbf{- F i g . ~ 5 ) . ~ A ~ s m a l l ~ v e i n ~ m a y ~ b e ~}$ found running near the bottom of the paravesical space, which should be sealed and cut.

Retractor blades are placed in the paravesical and pararectal spaces, and the blades are pulled medially. Caution should be taken not to scratch the sacral surface with a retractor blade. Pelvic lymphadenectomy can be performed when the upward (ventral) traction of the uterus and medial

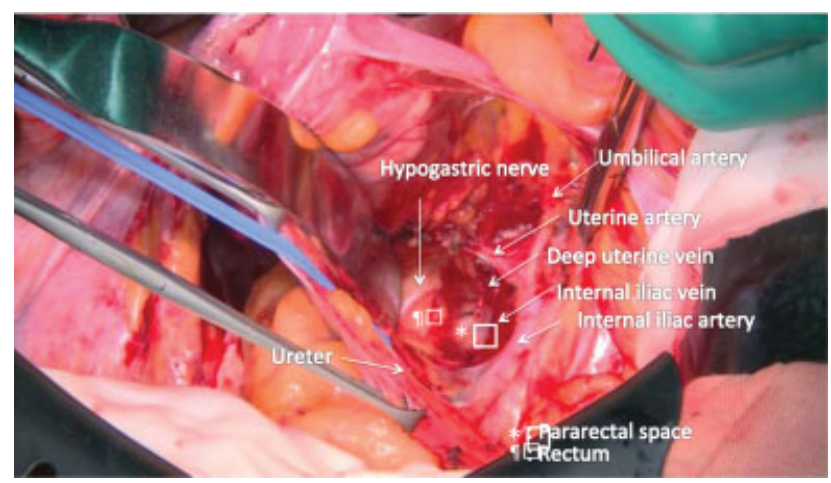

Fig. 4 Pararectal space (Latzko space) created by separating the tissue plane between the internal iliac artery/vein and the rectum. 


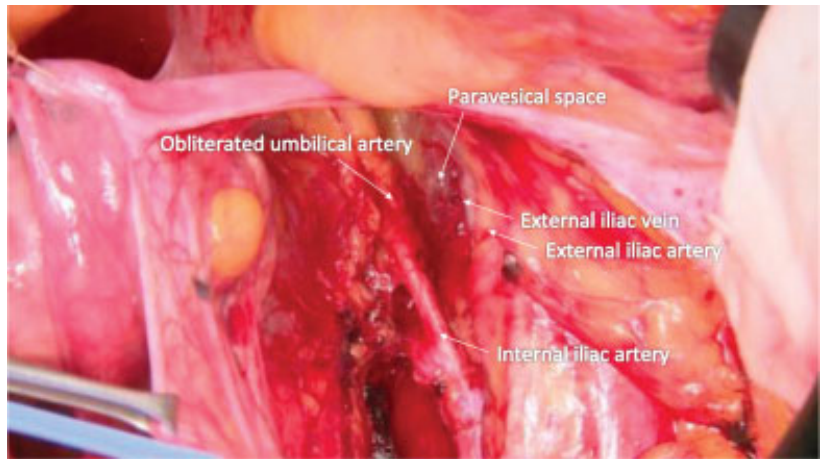

Fig. 5 Paravesical space created by separating the tissue plane between the umbilical artery and the external iliac artery/vein.

traction of the rectum and bladder using retractor blades are achieved.

\section{Pelvic Lymphadenectomy}

A thin membranous fascia (vascular sheath) encapsulates the arteries and veins. Lymphadenectomy is performed to remove the vascular sheath containing lymph nodes. The external iliac vessels and lymph nodes are separated from the psoas muscle medial to the genitofemoral nerve. Appropriate countertraction of the membranous fascia is needed. We then cut in the middle of the stretched membranous tissue, and the vascular sheath is peeled off from the vessels. A vessel loop is placed surrounding the external iliac artery, and gentle traction of the artery makes the tissue plane for dissection between the external iliac artery and vein. The vascular sheath of the external iliac artery and vein is incised. The external iliac vessel is bluntly and gently pushed away from the vascular sheath to remove the lymph nodes (-Fig. 6). Clear images of the topographic vascular anatomy are required for both safe and thorough lymphadenectomy. We carefully proceed with the dissection to avoid injury to the obturator nerve, which is buried in the fatty tissue containing the lymph nodes. The lymph nodes are dissected thoroughly from the pelvic sidewall lateral to the common iliac artery and vein to the deep uterine vein. The postoperative risk of lower-limb lymphedema can be reduced by

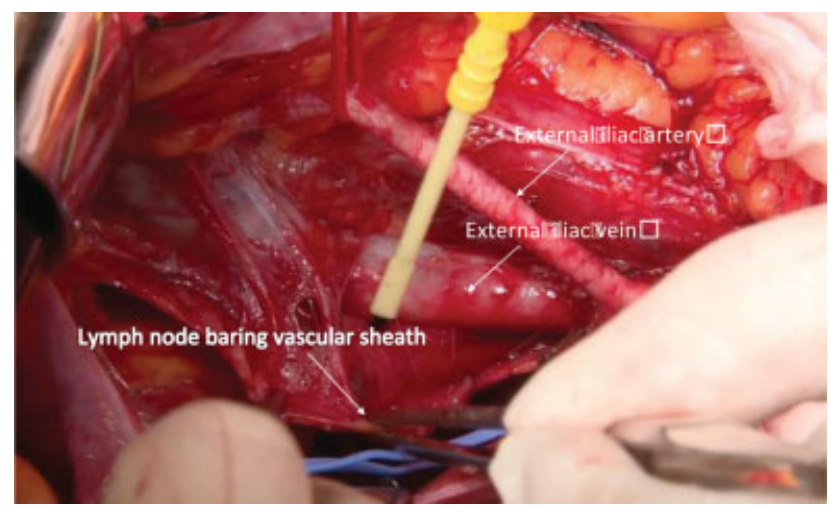

Fig. 6 Removing the lymph node baring vascular sheath of the external iliac vein in pelvic lymphadenectomy. The external iliac artery has been skeletonized.

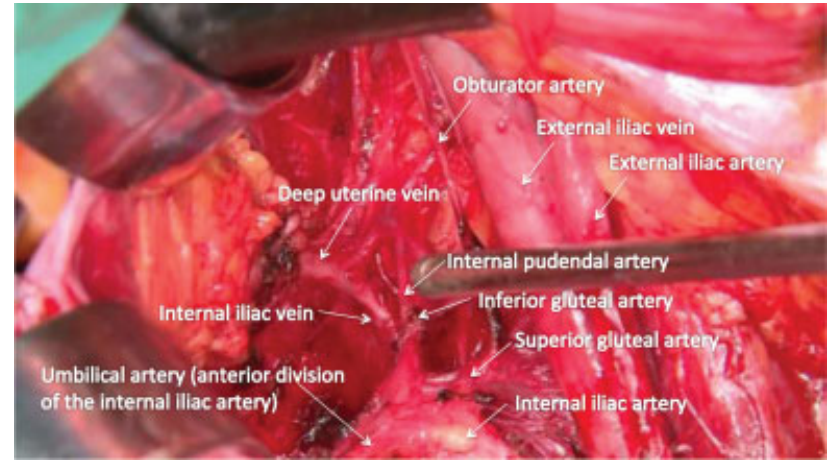

Fig. 7 The vascular structure in the lateral paracervix and the pelvic sidewall.

preserving the suprafemoral nodes (the circumflex iliac nodes distal to the iliac nodes). ${ }^{8}$ Metastasis to this nodal site is quite rare. ${ }^{9}$

After completion of lymphadenectomy, the intricate vascular network in the lateral paracervix and the pelvic sidewall is exposed (-Fig. 7). The hypogastric nerve is identified and can be taped, so it is recognizable during the following surgical procedure. On the pelvic sidewall, we can see skeletonized internal iliac and the deep uterine veins. The pelvic splanchnic nerves arise vertically from the anterior surface of the sacrum but not from the pelvic sidewall (-Fig. 8).

\section{Separation of the Bladder and Preparation of the Uterine Artery}

The bladder is separated from the cervix and the vagina. The separation of the bladder should be performed in the middle first and then extended laterally. The bladder should be separated cautiously to avoid damage to the small veins in the vesicouterine ligament (- Fig. 9).

\section{Removing the Lymph Node Baring Tissue around the Uterine Artery and the Cardinal Ligament Nodes}

This step is the beginning of the paracervical tissue dissection. The obliterated umbilical artery is held while the soft

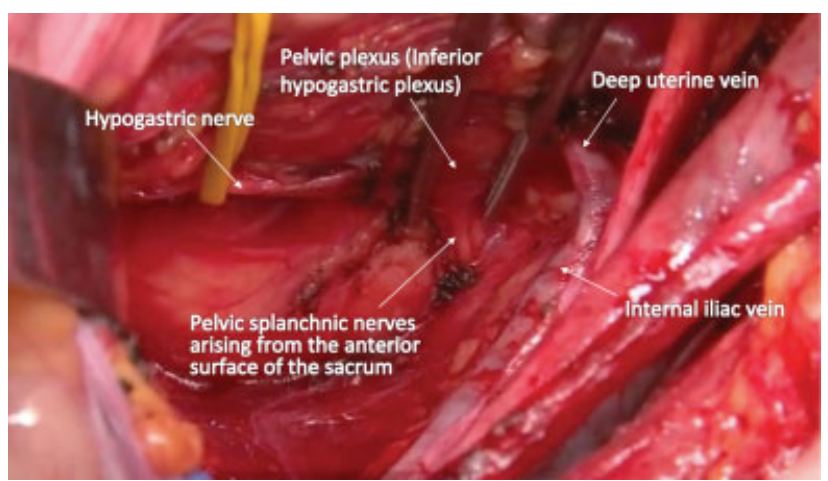

Fig. 8 The skeletonized internal iliac vein, the deep uterine vein, and the pelvic autonomic nerves. The deep uterine vein comprises the vascular portion of the cardinal ligament. The pelvic splanchnic nerves, which form the neural part of the cardinal ligament, arise from the sacrum's anterior surface but not from the pelvic sidewall. 


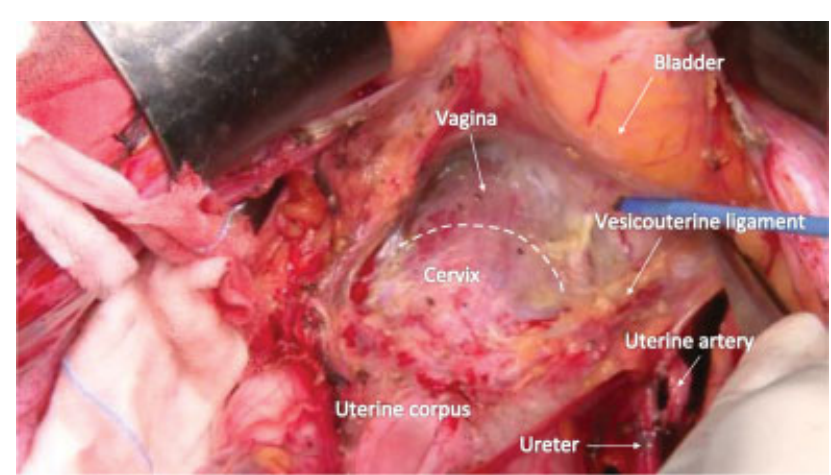

Fig. 9 The bladder is being separated from the vagina, leaving the vascular-rich portion of the vesicouterine ligament.

tissues, which may contain small lymph nodes around the uterine artery and the superficial uterine vein, are removed. The superficial uterine vein is transected. Lymph nodes around the deep uterine vein and the internal iliac vein are carefully and thoroughly removed.

\section{Separation of the Ureter and Transection of the Uterine Artery}

The dissection of the lateral paracervix proceeds further and the ureter is separated from the posterior leaf of the broad ligament and the uterosacral ligament. The thin membranous fascia encapsulating the ureter should be preserved without damage. The encapsulated ureter should be held using soft tape or the Penrose drain. The uterine artery is then transected medial to the ureter to keep the ureteral branch of the uterine artery for stage IB disease. For stage II disease, the uterine artery is transected at the origin from the internal iliac artery. With adequate traction of the ureter laterally, a small dimple can be found between the ureter and the uterine cervix; this is the entrance of the so-called ureteral tunnel. Kelly clamps are placed at the dimple, and the clamps are opened to push the ureter laterally by the clamps' outer blade ( $\boldsymbol{- F i g . 1 0}$ ). The widening of the tunnel proceeds steadily, taking the curve of the ureteral traveling path into account. The bladder is further separated cautiously to avoid bleeding from the vesicouterine ligament.

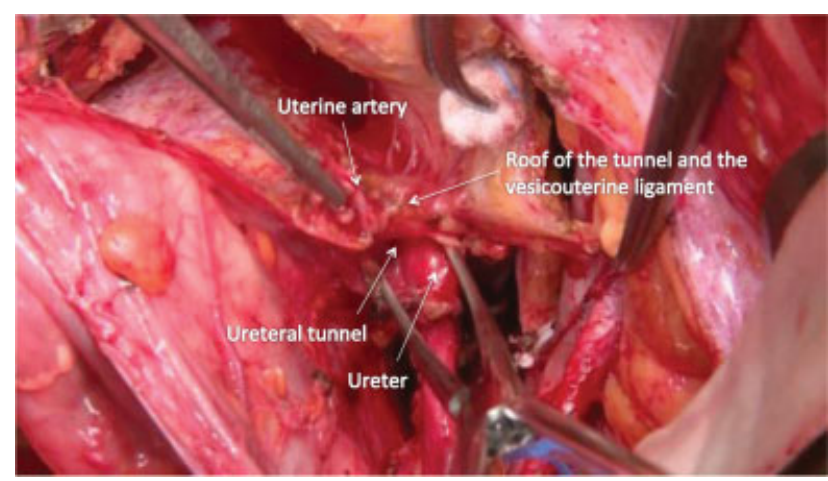

Fig. 10 The cut-end of the uterine artery is lifted upward, and the ureteral branch of the uterine artery is cut and ligated. The entrance of the ureteral tunnel is identified.

\section{Dissection and Transection of the Vesicouterine Ligament}

The vesicouterine and vesicovaginal ligaments are excised, an essential step in the Okabayashi-Kobayashi RH method. The ureteral tunnel is widely developed, and the ureter is pushed laterally and guarded using a Cooper scissors. Kelly clamps are introduced in the ureteral tunnel, and then the vesicouterine ligament is dissected and transected (- Fig. 11). Confirmation is needed to ensure that the ureter is not accidentally clamped or inappropriately close to the clamps.

\section{Separation of the Hypogastric Nerve and Transection of the Uterosacral Ligament}

The cul de sac peritoneum is incised, and the rectovaginal space is opened. Proper cranial traction of the rectum is essential to perform this procedure. The uterosacral ligament is transected to the level of the rectum. This procedure facilitates the mobility of the uterus, which will make the dissection of the vesicovaginal ligament easier. The hypogastric nerve is located and separated from the rectovaginal ligament for nerve-sparing $\mathrm{RH}$.

\section{Dissection and Transection of the Vesicovaginal Ligament}

The ureter is moved further laterally to expose the ves-

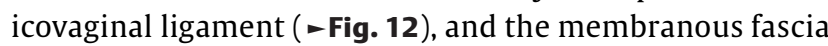
covering the surface of the vesicovaginal ligament is incised carefully to reveal the vesical veins and avoid bleeding. The cervicovesical vessels are transected when identified. The cranial part of the vesicovaginal ligament contains a vein from the ureter, which is isolated and transected. Then, the vesical veins in the main body of the vesicovaginal ligament are dissected. The vesicovaginal ligament is not an actual ligamentous tissue but rather composed of the vesical veins embedded in the fatty connective tissue and the vesical nerve fibers on the posterolateral surface. The vesical veins drain into the deep uterine vein (-Fig. 13). Thus, the vesical veins are observed between the bladder and the deep uterine vein (-Fig. 14, fresh cadaver dissection). Each vesical vein is isolated and transected.

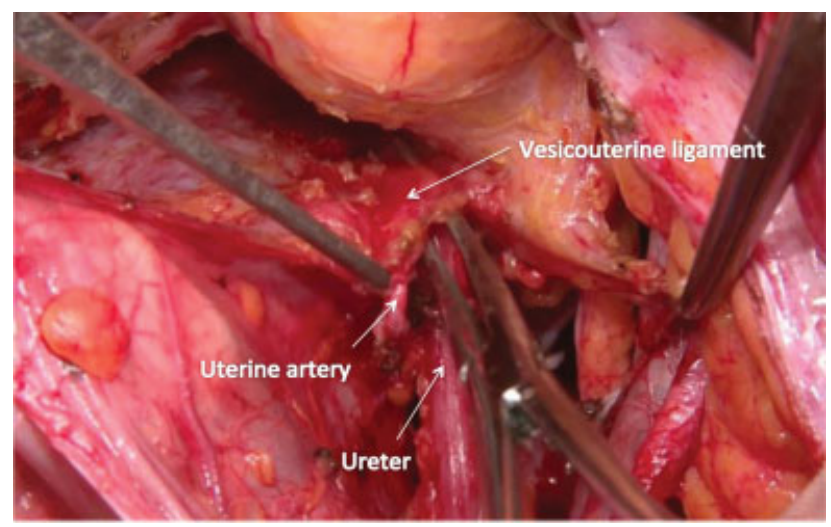

Fig. 11 The ureter is pushed laterally, and the vesicouterine ligament is transected. 


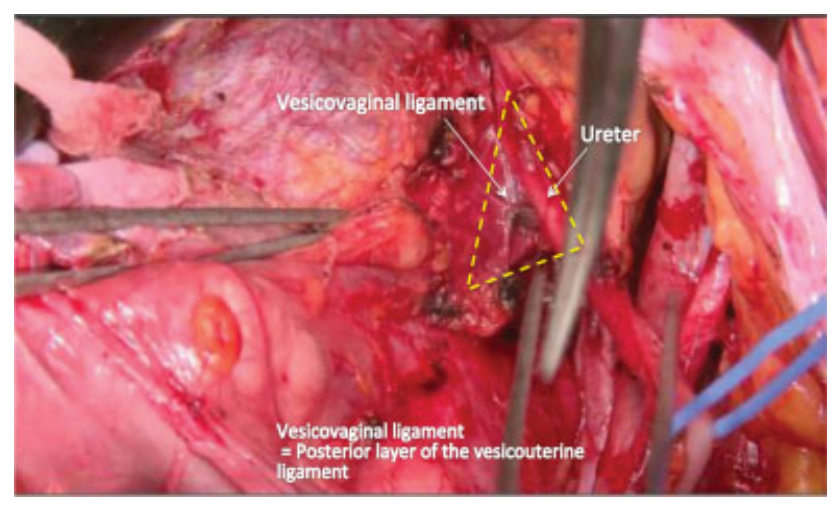

Fig. 12 The ureter is pushed laterally using a cotton peanut swab to expose the vesicovaginal ligament widely. We can recognize a thin membranous connective tissue covering the vesicovaginal ligament and identify the vesical veins by removing the membranous fascia.

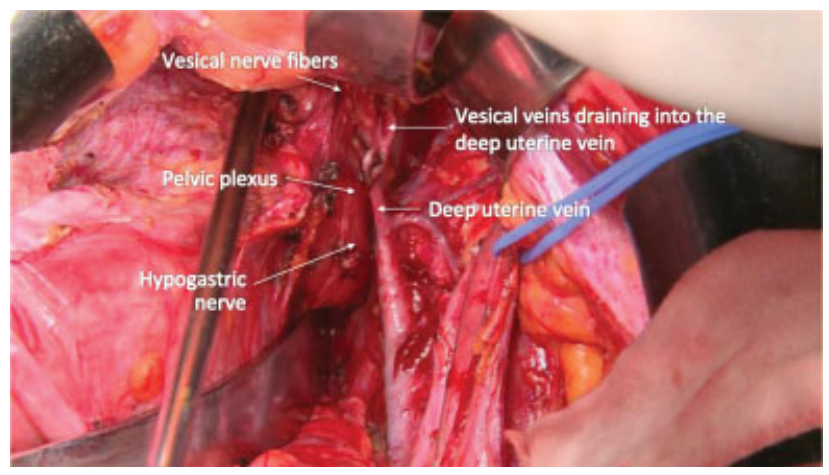

Fig. 13 By retracting the ureter caudally, we can see the vesical veins draining into the deep uterine vein.

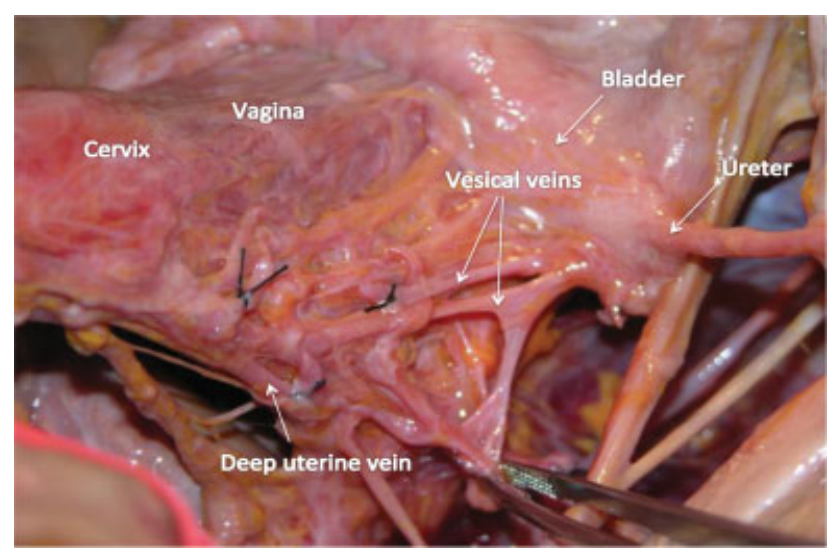

Fig. 14 A fresh cadaver dissection shows two vesical veins from the bladder to the deep uterine vein.

\section{Transection of the Deep Uterine Vein (Vascular Portion of the Cardinal Ligament)}

The deep uterine vein is skeletonized and transected (-Fig. 15). The cut-end of the deep uterine vein of the uterine side is clamped and then separated bluntly from the inferior hypogastric plexus, using a cotton peanut swab. If an additional vesical vein is identified, it is also transected. There is a small vein penetrating the inferior hypogastric plexus to be

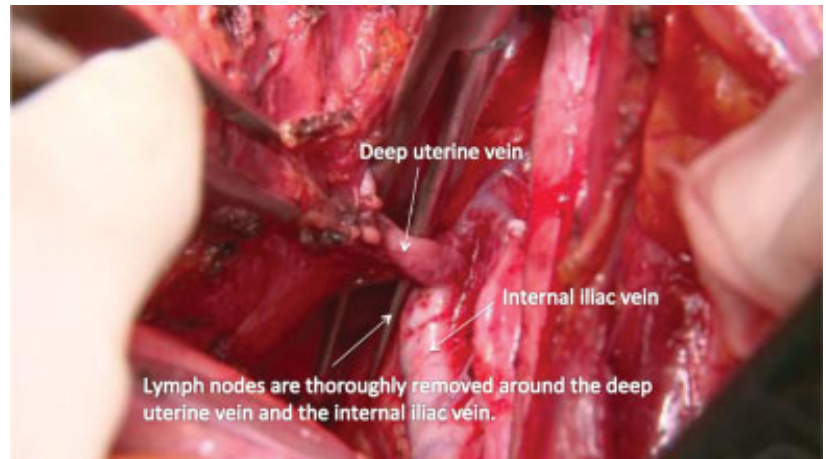

Fig. 15 The deep uterine vein, the vascular portion of the cardinal ligament, is cut at the origin from the internal iliac vein.

transected. After sufficient separation and upward traction of the deep uterine vein, the pelvic plexus is identified on the cervix's lateral aspect and the upper vagina.

\section{Selective Transection of the Uterine Branches of the Pelvic Plexus (Inferior Hypogastric Plexus) and Preservation of the Pelvic Plexus and Its Vesical Branches}

The integrity of both the sympathetic and parasympathetic nerve supply is necessary for maintaining bladder function. We need to preserve the hypogastric nerve, the pelvic splanchnic nerves, the pelvic plexus, and the vesical nerve branches of the pelvic plexus. ${ }^{6}$ We locate the uterine nerve branches and vesical nerve branches from the posterior and anterior (-Fig. 16, fresh cadaver dissection). The cut-end of the deep uterine vein (vascular portion of the cardinal ligament) is rubbed off the pelvic splanchnic nerves and the inferior hypogastric plexus (-Fig. 17). The vesical nerve fibers can be recognized on the upper lateral surface of the paracolpium. However, it is often difficult to identify the vesical nerve fibers visually. The uterine nerve fibers can serve as the landmark of the vesical nerve fibers. The pelvic plexus and the uterine nerve fibers are separated from the paracervix. The uterine nerve fibers will be selectively transected in the nerve-sparing $\mathrm{RH}$ to detach the nervous system

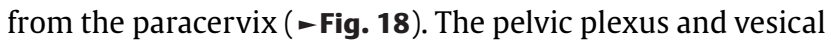

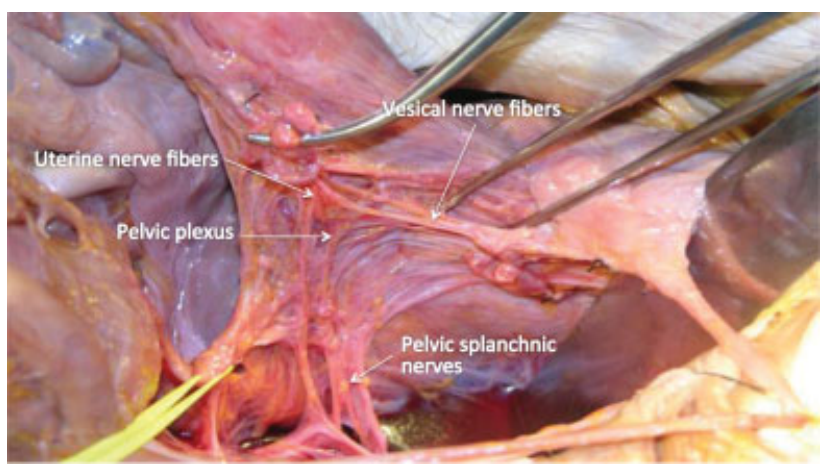

Fig. 16 A fresh cadaver dissection illustrates that we can identify the uterine nerve branches of the pelvic plexus by detaching the deep uterine vein from the pelvic plexus gently, using a cotton peanut swab. 


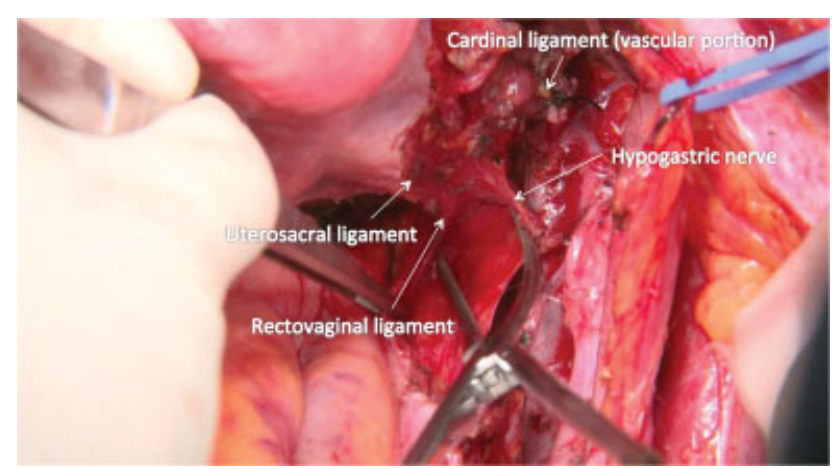

Fig. 17 After the cut-end of the vascular portion of the cardinal ligament is pulled upward and separated from the pelvic plexus, we divide the hypogastric nerve from the rectovaginal ligament. The separation should proceed up to the uterine nerve fibers.

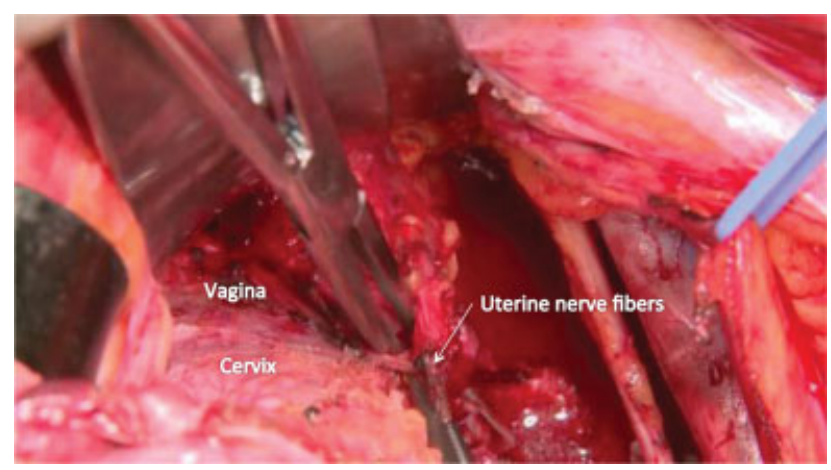

Fig. 18 The uterine nerve fibers are separated from the paracervix with caution not to dissect the wrong tissue plane containing a part of the paracervix.

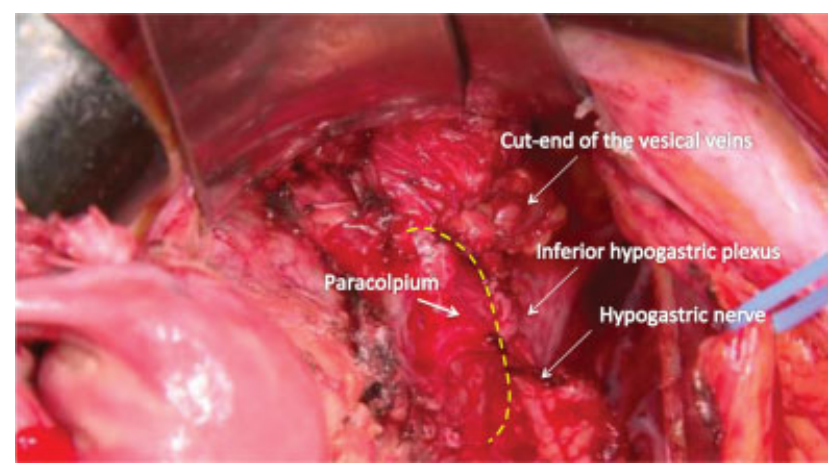

Fig. 19 The nerve plate composed of the pelvic plexus, hypogastric nerve, and the vesical nerve fibers moves dorsolaterally, leaving the paracolpium, which has to be excised.

nerve fibers move posterolaterally away from the paracolpium (-Fig. 19).

In a non-nerve-sparing $\mathrm{RH}$, the dissection method for the vesicovaginal ligament is different. There is a triangular zone formed by the bladder, the vagina and paracolpium, and the vesicovaginal ligament. With a finger placed behind the vesicovaginal ligament and pushing the bladder downward and the ureter laterally, we can find an avascular area lateral to the paracolpium. This point is the entrance of the paravaginal space, which connects the paravesical space. The paravaginal space is opened to dissect the vesicovaginal ligament in a non-nerve-sparing $\mathrm{RH}$. In a nerve-sparing $\mathrm{RH}$, we do not develop the paravaginal space.

\section{Transection of the Remaining Rectovaginal Ligament} The rectum is pushed downward for resection of the rectovaginal ligament. We transect the rectovaginal ligament as far as the vagina and then suture-ligate the paracolpium, and the vaginal excision is performed.

\section{Amputation of the Vagina/Paracolpium and Suture}

The paracolpium is clamped and doubly suture-ligated using Vicryl 1-0 suture. Caution should be taken not to damage the preserved nerve structures. The paracolpium and vagina are amputated using bipolar scissors. The vaginal cuff may be suture closed, although this will cause vaginal shortening. Since most patients who undergo a $\mathrm{RH}$ are at a younger age, we prefer the method of leaving the vaginal stump open. In the open method, we use U-shaped sutures for the vaginal wall. The suture is introduced from the inside to the outside and then returned from the outside to the inside ( - Figs. 20, 21). The string is tied inside the vagina, which will be removed 7 to 10 days after the surgery. The vaginal stump is covered by suturing the bladder peritoneum and the rectum peritoneum (-Fig. 22) ${ }^{7,10}$

\section{Dissection of the Common Iliac Nodes and Presacral Nodes}

The common iliac nodes and presacral lymph nodes are dissected. It is our practice to perform lymphadenectomy, including these lymph node groups, unless the tumor is small $(<2 \mathrm{~cm})$ and without prominent lymphovascular space invasion.

\section{Ovarian Transposition}

This procedure is indicated for younger cervical cancer patients. The ovaries are transposed to the paracolic gutters to protect them from the radiation that might be indicated

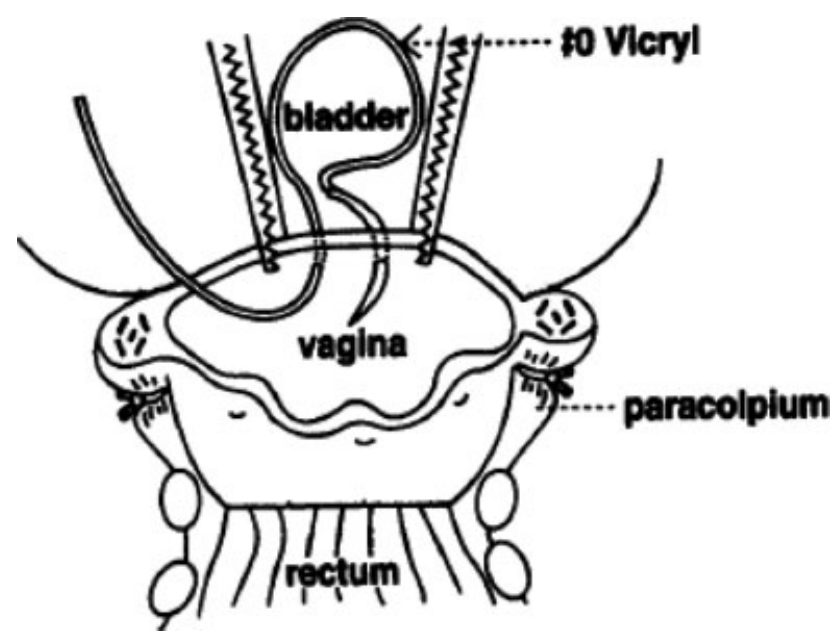

Fig. 20 The open method of vaginal stump management to prevent vaginal shortening after radical hysterectomy $(\mathrm{RH})$. 


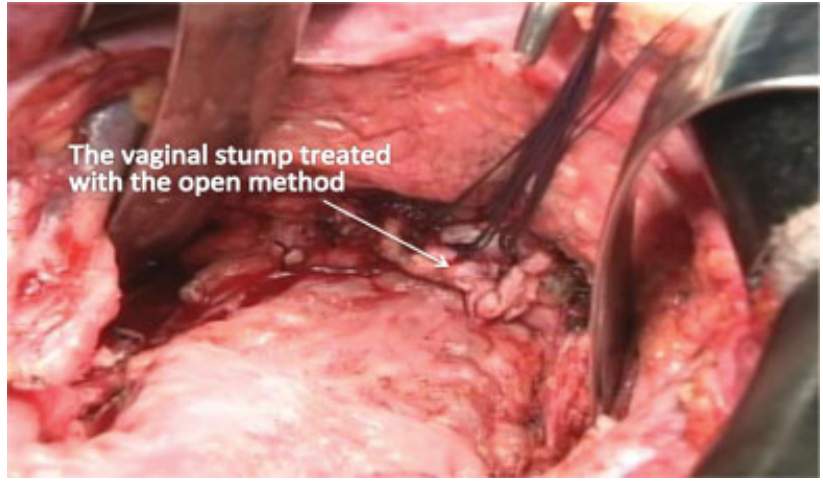

Fig. 21 The vaginal stump is left open using inverted U-shape sutures to prevent vaginal shortening.

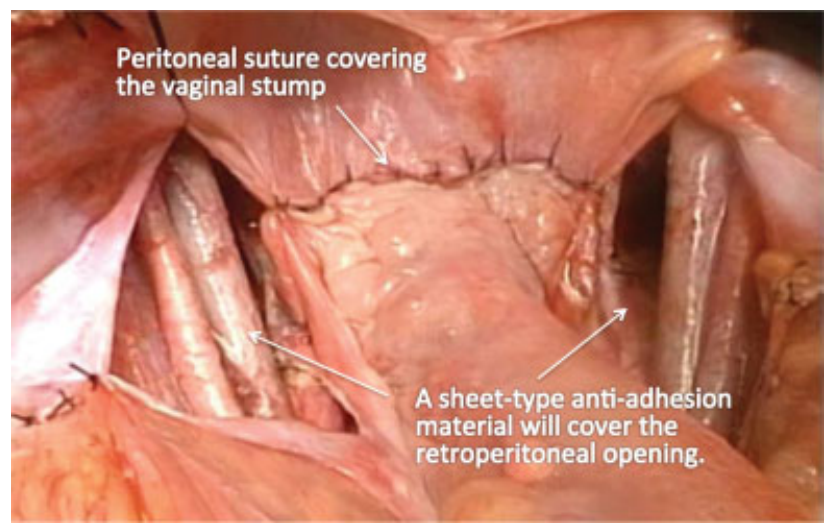

Fig. 22 We cover the open vaginal vault by suturing the bladder peritoneum and the rectum peritoneum.

postoperatively and to avoid ovarian cyst or pseudocyst formation due to adhesion around the ovaries left in the pelvis. $^{7}$

\section{Peritoneal Closure}

The pelvic peritoneum is left open except the covering area of the vaginal stump. It is essential to perform some preventive measures for the adhesion of the small bowel in the bottom of the pelvis. We place antiadhesion material on the peritoneal surface of the pelvis. We also use rectosigmoid that was mobilized for lymphadenectomy to fill the pelvic cavity, so the small intestine will not fall into the pelvic cavity.

\section{Status of the Pelvic Cavity after Nerve-Sparing Radical Hysterectomy}

We can preserve a fence-like structure of the pelvic autonomic nervous system after the completion of nerve-sparing RH ( - Fig. 23). The T-shaped pelvic nerve sheet is preserved (-Fig. 24, fresh cadaver dissection).

\section{Abdominal Closure}

For closure of the abdomen, we prefer and recommend the Smead-Jones single mass closure method with the absorb-

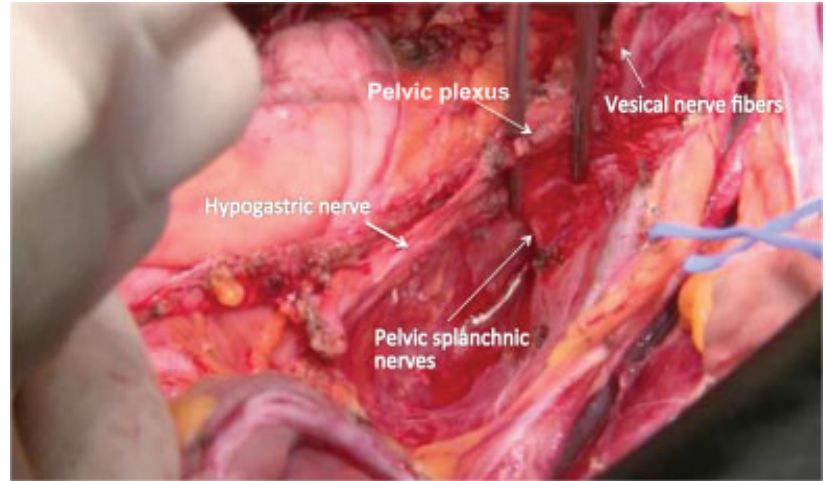

Fig. 23 The pelvic autonomic nerves preserved totally after the systematic nerve-sparing radical hysterectomy $(\mathrm{RH})$.

able sutures (Vicryl 1-0 or PDS-II 1-0) instead of layer-bylayer closure.

\section{Tailored Radical Hysterectomy for Locally Advanced Stage Cervical Cancer}

The extent of the paracervical tissue and the vaginal excision should be tailored to the disease stage. ${ }^{11}$ We compare the area of the paracervical tissue removal and the management of the pelvic autonomic nerves among various methods of $\mathrm{RH}$. For stage IB disease, we use the systematic nerve-sparing RH. A fresh cadaver dissection demonstrates how the nervesparing method works ( - Fig. 25). For stage II disease, we use a partial nerve-sparing $\mathrm{RH}$ technique that preserves the pelvic splanchnic nerves and a part of the pelvic plexus using the Kobayashi method (Line $\mathrm{K}$ in -Fig. 26). In rare instances, we extirpate the whole pelvic autonomic nerves around the uterine cervix to remove the tumor extending to the paracervix using the original Okabayashi method (Line $\mathrm{O}$ in - Fig. 26). In our experience, we have obtained satisfactory oncological outcomes for locally advanced cervical cancer using the Okabayashi-Kobayashi $\mathrm{RH}$ without adjuvant radiotherapy. ${ }^{12,13}$

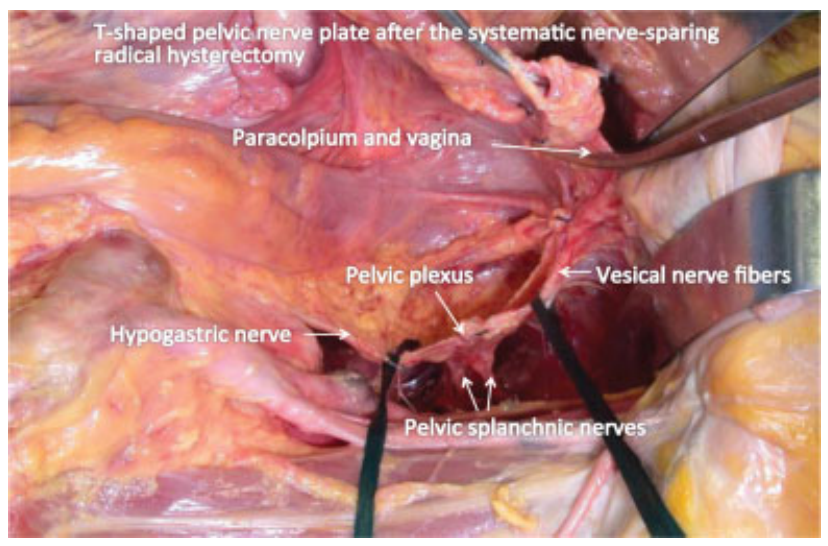

Fig. 24 The T-shaped pelvic nerve sheet is preserved (fresh cadaver dissection). 


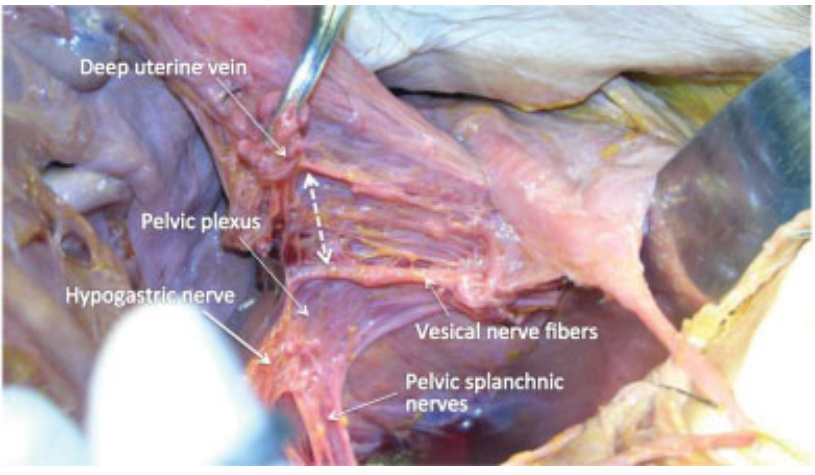

Fig. 25 Paracervical tissue and the vagina/paracolpium excision in a systematic nerve-sparing radical hysterectomy $(\mathrm{RH})$.

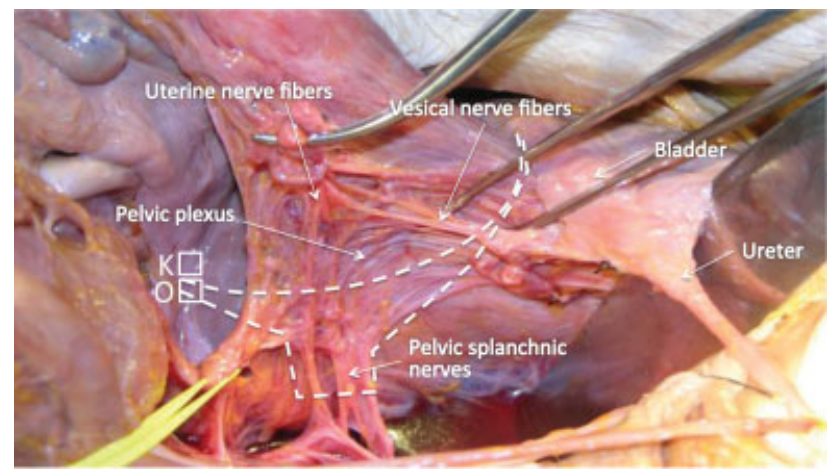

Fig. 26 Radical hysterectomy (RH) for stage II disease. The Kobayashi method (line K) preserves the pelvic splanchnic nerves and a part of the pelvic plexus and its vesical branches. The original Okabayashi method (line $\mathrm{O}$ ) removes paracervical tissue extensively, including the pelvic splanchnic nerves and the pelvic plexus.

\section{Conflict of Interest}

None declared.

\section{References}

1 Meigs JV. Radical hysterectomy with bilateral pelvic lymph node dissections; a report of 100 patients operated on five or more years ago. Am J Obstet Gynecol 1951;62(04):854-870

2 Okabayashi H. Radical abdominal hysterectomy for cancer of the cervix uteri. Modification of the Takayama operation. Surg Gynecol Obstet 1921;33:335-341

3 Latzko W, Schiffmann J. Klinisches und Anatomisches zur Radikaloperation des Gebarmutterkrebses. Zentralbl Gynäkol 1919; 43:715-719

4 Kobayashi T. Abdominal Radical Hysterectomy With Pelvic Lymphadenectomy for Cancer of the Cervix. Tokyo: Nanazando1961

5 Sakamoto S, Takizawa K. An improved radical hysterectomy with fewer urological complications and with no loss of therapeutic results for invasive cervical cancer. Baillieres Clin Obstet Gynaecol 1988;2(04):953-962

6 Sakuragi N, Todo Y, Kudo M, Yamamoto R, Sato T. A systematic nerve-sparing radical hysterectomy technique in invasive cervical cancer for preserving postsurgical bladder function. Int J Gynecol Cancer 2005;15(02):389-397

7 Kaneuchi M. Radical hysterectomy (function-preserving method). In: Sakuragi N, ed. Surgery for Cervical Cancer and Vulvar Cancer. OGS Now No. 5. Tokyo: Medical View Co. Ltd; 2010

8 Ohba Y, Todo Y, Kobayashi N, et al. Risk factors for lower-limb lymphedema after surgery for cervical cancer. Int J Clin Oncol 2011;16(03):238-243

9 Sakuragi N, Satoh C, Takeda N, et al. Incidence and distribution pattern of pelvic and paraaortic lymph node metastasis in patients with Stages IB, IIA, and IIB cervical carcinoma treated with radical hysterectomy. Cancer 1999;85(07):1547-1554

10 Yamamoto R, Okamoto K, Ebina Y, Shirato H, Sakuragi N, Fujimoto $\mathrm{S}$. Prevention of vaginal shortening following radical hysterectomy. BJOG 2000;107(07):841-845

11 Sakuragi N, Murakami G, Konno Y, Kaneuchi M, Watari H. Nervesparing radical hysterectomy in the precision surgery for cervical cancer. J Gynecol Oncol 2020;31(03):e49

12 Sakuragi N, Kato T, Shimada C, et al. Oncological outcomes after Okabayashi-Kobayashi radical hysterectomy for early and locally advanced cervical cancer. JAMA Netw Open 2020;3(05):e204307

13 Sakuragi N, Kaneuchi M, Kato T, et al. Tailored radical hysterectomy for locally advanced cervical cancer. Int J Gynecol Cancer 2020; 30(08):1136-1142 